\title{
Chemical Composition Similarity Relationships among the Various Organs of the Ilex cornuta Lindl. \& Paxton Based on the Analysis of Hydrophilic Volatile Compounds
}

\author{
Luosheng Huang* \\ School of Traditional Chinese Pharmacy, China Pharmaceutical University, Nanjing 211198, P.R.China
}

${ }^{*}$ Corresponding author: Luosheng Huang, School of Traditional Chinese Pharmacy, China Pharmaceutical University, Nanjing 211198, P.R.China; Tel: +86 13505154540; E-mail: huangluosheng@hotmail.com

Received: March 31, 2021; Accepted: April 07, 2021; Published: April 10, 2021

\begin{abstract}
In performing molecular profiling of secondary metabolites, a lot of research has focused on biogenic volatile organic compounds with medium to low polarity. In this study, chemical composition similarity relationships among the various organs of the Ilex cornuta Lindl. \& Paxton were assessed based on the analysis of hydrophilic volatile compounds. GC-MS analysis was conducted to characterize and classify the chemical compounds. A total of 36, 46, 42, 25, 64, 26 compounds have been respectively extracted from the root, stem, stem skin, leaf, flower and fruit. The six organs have 3 common compounds and large percentages of exclusive compounds ranging from $36.0 \%$ to $62.5 \%$ with a mean of $49.8 \%$, indicating substantial component differences among the different organs. The percentage of overlapping compounds between each of the two organs ranges from $10.9 \%$ to $44.0 \%$, which is relatively small, further demonstrating the strong organ specificity of the chemical composition. The overlapping index is used to reveal the similarity among the organs. The stem shares the maximum similarity while the fruit the minimum similarity with the other organs. Aside from fruit, the average overlapping indices between each of the other two organs correlate well to their physical proximity. In conclusion, hydrophilic volatile metabolites are a class of natural products that are rarely investigated but constitute a significant part of the plant chemical composition. Chemical profiling of these metabolites could provide a valuable tool for the plant taxonomy and help understand the chemically mediated biological phenomena.
\end{abstract}

Keywords: Chemical composition similarity, GC-MS, Hydrophilic volatile compounds, Ilex cornuta Lindl. \& Paxton, Plant taxonomy

\section{Introduction}

Plant taxonomy is traditionally conducted based on macroscopic and microscopic morphological characteristics. Growing evidence suggests that many biologically relevant entities could be missed in the studies that rely solely on morphological traits, particularly since speciation is not always accompanied by morphological change $[1,2]$. In recent years, plant chemical taxonomy has been developed to perform classification based on a wide array of biologically active secondary metabolites [3]. The expression of secondary metabolites could vary due to convergent evolution or differential gene expression [4], suggesting that the metabolite content of plants may reveal more information on the bioactive pattern of plants in comparison to morphology characterization [5].

In performing molecular profiling of secondary metabolites, a lot of research has focused on biogenic volatile organic compounds with medium to low polarity [6-9]. Volatile compounds are secreted and part of them are volatilized immediately after secretion $[10,11]$. The remaining part is stored in the special structure of the plant as in the case of essential oils [12-14]. Additionally, Berlinck and collaborators found that the vast majority of new compounds from natural sources reported in recent literature are compounds of medium to low polarity. Water-soluble, volatile, minor and photosensitive natural products are yet poorly known. One of the possible reasons for this trend could be that organic solvents of medium to low polarity used in isolation procedures require less time and less sophisticated instrumentation to be evaporated [15]. The author speculates that there is a class of hydrophilic volatile compounds in plants that are dispersed or dissolved in the water phase, evaporated with water vapor, and whose polarity and volatility are somewhere between essential oils and watersoluble compounds. To protect this type of ingredients from loss during extraction, water vapor distillation is used to collect volatile compounds that are dispersed or dissolved in the plant's water phase. The root, stem, stem skin, leaf, flower and fruit of the Ilex cornuta Lindl. \& Paxton were analyzed as study samples. Volatile essential oils were removed by using Soxhlet extraction method. Hydrophilic volatile compounds obtained by water reflux extraction are characterized and classified by quantitative GC-MS. The study revealed the potential use of hydrophilic volatile metabolites in the plant taxonomy and understanding the chemically mediated biological phenomena. 


\section{Materials and Methods}

\section{Material}

Ilex cornuta Lindl. \& Paxton was collected in Nanjing, China. Its roots, stems, stem skins, leaves, flowers and fruits were washed, cut into pieces, dried at $30^{\circ} \mathrm{C}$ and stored at $2-8^{\circ} \mathrm{C}$ prior to use.

\section{Chemicals and Reagents}

Ethyl acetate was purchased from Xilong Chemical Co., Ltd (Shantou, China). Hexane was purchased from Shanghai Titan Scientific Co., Ltd (Shanghai, China). Activated carbon was purchased from Shanghai Chemical Reagent Procurement Center (Shanghai, China). C7-C40 saturated alkanes standard was purchased from Anpel Laboratory Technologies Inc. (Shanghai, China).

\section{Sample Preparation}

Each sample was sliced and dried at $30^{\circ} \mathrm{C}$. After ground into powder, the samples were sieved through 80 mesh followed by 180 mesh. Approximately $6 \mathrm{~g}$ of the sample were subjected to Soxhlet extractor method with hexane for 24 hrs to remove essential oils and other lipophilic compounds. The remainder was then removed and dried at $30^{\circ} \mathrm{C}$ in the ventilation cabinet. Approximately $4 \mathrm{~g}$ of the dried powder was then added into a $6 \times 7 \mathrm{~cm}$ nonwoven bag together with three glass balls of $4 \mathrm{~cm}$ diameter. At least 3 segments of thread were used to separate and tighten the bag into 3 parts, each containing a glass ball and even amount of the dried powder. The bag was then placed in a flask and $2100 \mathrm{~mL}$ of water was subsequently added to soak the powder for about $2 \mathrm{hrs}$. After reflux extraction for $6 \mathrm{hrs}, 2 \mathrm{~L}$ of distilled water was collected. The same reflux extraction was repeated to collect another $1 \mathrm{~L}$ of distilled water for a total of $3 \mathrm{~L}$. After cooling, activated carbon (4 g) was added to absorb the active ingredients from the $3 \mathrm{~L}$ of distilled water for about $8 \mathrm{hrs}$. The activated carbon containing the active ingredients was then filtered and dried at $30^{\circ} \mathrm{C}$ for $12 \mathrm{hrs}$. Ethyl acetate was subsequently added to isolate the active ingredients from the activated carbon using Soxhlet extractor method for $8 \mathrm{hrs}$. The resulting ethyl acetate extract was left in the ventilation cabinet to dry at $30^{\circ} \mathrm{C}$. The dried active ingredients were finally re-dissolved using ethyl acetate, filtrated through $0.22 \mu \mathrm{m}$ filter and analyzed using GC-MS.

\section{GC-MS Analysis}

Analysis of hydrophilic volatile compounds was performed using Shimadzu GCMS-QP2010 Single Quadrupole GC-MS (Kyoto, Japan). A Rxi-1 ms GC capillary column $(30 \mathrm{~cm}$ length, $0.25 \mathrm{~mm}$ inner diameter and $0.25 \mu \mathrm{m}$ thick film) from Shimadzu (Kyoto, Japan) was used for analysis.

One microliter of sample was injected in split mode with split ratio of 5 to 1 . GC inlet temperature is set at $280^{\circ} \mathrm{C}$. High purity nitrogen $(\geq 99.999 \%)$ was used as carrier gas in constant flow mode at $1 \mathrm{~mL} / \mathrm{min}$. The initial temperature of the $\mathrm{GC}$ oven is set at $60^{\circ} \mathrm{C}$ and held for $1 \mathrm{~min}$, then ramped at $4^{\circ} \mathrm{C} / \mathrm{min}$ to $160^{\circ} \mathrm{C}$ and held for 3 mins, followed by $2^{\circ} \mathrm{C} / \mathrm{min}$ to $280^{\circ} \mathrm{C}$ and held for 6 mins. Finally, the temperature is raised to $300^{\circ} \mathrm{C}$ at $4^{\circ} \mathrm{C} / \mathrm{min}$ and held for 6 mins. The mass spectrometer was operated in positive electron ionization mode at $70 \mathrm{eV}$ and all spectra were recorded in full scan with a mass range of $40-700 \mathrm{Da}$. The interface temperature is set at $280^{\circ} \mathrm{C}$ and ion source temperature is set at $250^{\circ} \mathrm{C}$.

\section{Data Processing and Compound Identification}

The GC-MS data processing was done with Shimazdzu GCMS Solution software. Compound identification was performed by applying several assignments, e.g., reference standard analysis, retention index calculation, and by NIST08 Spectrum Library comparison. Only peaks with area greater than 3 million are analyzed. The overlapping percentage is calculated by the number of overlapping compounds divided by the total number of hydrophilic volatile compounds from each of the two organ and times 100. Overlapping index is calculated by the number of overlapping compounds squared and divided by the total number of hydrophilic volatile compounds from each of the two organs. In addition, hierarchical clustering analysis was performed with Python to assess the similarities between each of the two organs by analyzing the number of overlapping hydrophilic volatile compounds.

\section{Results and Discussion}

The root, stem, stem skin, leaf, flower and fruit of the Ilex cornuta Lindl. \& Paxton contain compounds that are water soluble and can volatilize with water vapor. These hydrophilic compounds do not separate from the water phase and possess greater polarity than essential oils. The largest number (64) of hydrophilic volatile compounds are isolated from the flower and the smallest (25) from the leaf, indicating that the number of hydrophilic volatile compounds varies greatly from organ to organ. The hydrophilic volatile compounds include aromatics, fatty acids, furans, heterocycle, esters, alkanes, ketones, halogens and other types of small molecular compounds. This is a diverse group of molecules that could contribute to the expression of biological information about the plant. Tables 1-6 present the lists of hydrophilic volatile compounds identified from the root, stem, stem skin, leaf, flower and fruit, respectively. The bold and italic fonts in the table are used to refer to exclusive compounds that are only found in the specific organ and not contained in any other organ.

As shown in Table 7, the total number of hydrophilic volatile compounds isolated from the six organs ranges from 25 to 64 . There are 3 common compounds in the six organs, i.e. Dodecanoic acid, 1,2-Benzenedicarboxylic acid, bis(2-methylpropyl) ester and n-Hexadecanoic acid. This accounts for $12.0 \%$ of total hydrophilic volatile compounds for the leaf and $4.7 \%$ for the flower with an average of $8.4 \%$ for all the six organs, indicating the little commonality of the six organs. Each organ also has its exclusive compounds which are not found in any other organ. The percentage of exclusive compounds follows the order of flower $>$ fruit $>$ stem skin $>$ root $>$ stem $>$ leaf. The flower has the largest number and percentage of the exclusive compounds, 40 and $62.5 \%$, respectively. The leaf has the smallest number and percentage of the exclusive compounds, 9 and $36.0 \%$, respectively. The stem and stem skin display medium numbers of exclusive compounds. The average percentage of the exclusive compounds in the six organs was $49.8 \%$, nearly half, indicating strong organ specificity. These results provide evidence to support the 
Luosheng Huang (2021) Chemical Composition Similarity Relationships among the Various Organs of the Ilex Cornuta Lindl. \& Paxton Based on the Analysis of Hydrophilic Volatile Compounds

Table 1: List of the hydrophilic volatile compounds identified from the root of the Ilex cornuta Lindl. \& Paxton.

\begin{tabular}{|c|c|c|c|c|}
\hline No & RT & RI & Compound & Formula \\
\hline 1 & 8.434 & 1041 & 2(3H)-Furanone, dihydro-4-hydroxy- & $\mathrm{C}_{4} \mathrm{H}_{6} \mathrm{O}_{3}$ \\
\hline 2 & 8.555 & 1044 & 2-Oxo-n-valeric acid & $\mathrm{C}_{5} \mathrm{H}_{8} \mathrm{O}_{3}$ \\
\hline 3 & 8.623 & 1047 & 2,3-Anhydro-d-galactosan & $\mathrm{C}_{6} \mathrm{H}_{8} \mathrm{O}_{4}$ \\
\hline 4 & 9.159 & 1064 & Acetic acid, hexyl ester & $\mathrm{C}_{8} \mathrm{H}_{16} \mathrm{O}_{2}$ \\
\hline 5 & 9.767 & 1084 & 2-Cyclopenten-1-one, 3-ethyl-2-hydroxy- & $\mathrm{C}_{7} \mathrm{H}_{10} \mathrm{O}_{2}$ \\
\hline 6 & 12.753 & 1173 & Octanoic Acid & $\mathrm{C}_{8} \mathrm{H}_{16} \mathrm{O}_{2}$ \\
\hline 7 & 13.662 & 1199 & 2-Furancarboxaldehyde,5-(hydroxymethyl)- & $\mathrm{C}_{6} \mathrm{H}_{6} \mathrm{O}_{3}$ \\
\hline 8 & 16.053 & 1269 & Nonanoic acid & $\mathrm{C}_{9} \mathrm{H}_{18} \mathrm{O}_{2}$ \\
\hline 9 & 19.301 & 1364 & Benzaldehyde, 4-(methylthio)- & $\mathrm{C}_{8} \mathrm{H}_{8} \mathrm{OS}$ \\
\hline 10 & 23.459 & 1492 & 1H-2-Benzopyran-1-one, 3,4-dihydro-8-hydroxy-3-methyl- & $\mathrm{C}_{10} \mathrm{H}_{10} \mathrm{O}_{3}$ \\
\hline 11 & 23.660 & 1498 & 3-Acetoxydodecane & $\mathrm{C}_{14} \mathrm{H}_{28} \mathrm{O}_{2}$ \\
\hline 12 & 25.161 & 1546 & 7-Hydroxy-3-(1,1-dimethylprop-2-enyl) coumarin & $\mathrm{C}_{14} \mathrm{H}_{14} \mathrm{O}_{3}$ \\
\hline 13 & 25.496 & 1557 & Dodecanoic acid & $\mathrm{C}_{12} \mathrm{H}_{24} \mathrm{O}_{2}$ \\
\hline 14 & 25.696 & 1564 & Estra-1,3,5(10)-trien-17. beta. - ol & $\mathrm{C}_{18} \mathrm{H}_{24} \mathrm{O}$ \\
\hline 15 & 26.109 & 1577 & Butyric acid, 3-tridecyl ester & $\mathrm{C}_{17} \mathrm{H}_{34} \mathrm{O}_{2}$ \\
\hline 16 & 26.829 & 1600 & Hexadecane & $\mathrm{C}_{16} \mathrm{H}_{34}$ \\
\hline 17 & 27.305 & 1613 & Ethanone, 1-[2-(5-hydroxy-1,1-dimethylhexyl)-3-methyl-2-cyclopropen-1-yl]- & $\mathrm{C}_{14} \mathrm{H}_{24} \mathrm{O}_{2}$ \\
\hline 18 & 28.020 & 1631 & Thieno[3,2-c]pyridin-4(5H)-one & $\mathrm{C}_{7} \mathrm{H}_{5} \mathrm{NOS}$ \\
\hline 19 & 28.671 & 1649 & Dodecanoic acid, 3-hydroxy- & $\mathrm{C}_{12} \mathrm{H}_{24} \mathrm{O}_{3}$ \\
\hline 20 & 30.636 & 1700 & 2-Bromotetradecane & $\mathrm{C}_{14} \mathrm{H}_{29} \mathrm{Br}$ \\
\hline 21 & 32.780 & 1750 & 7-Methyl-Z-tetradecen-1-ol acetate & $\mathrm{C}_{17} \mathrm{H}_{32} \mathrm{O}_{2}$ \\
\hline 22 & 35.860 & 1821 & 1,2-Benzenedicarboxylic acid, bis(2-methylpropyl) ester & $\mathrm{C}_{16} \mathrm{H}_{22} \mathrm{O}_{4}$ \\
\hline 23 & 37.894 & 1867 & $\begin{array}{l}\text { 2a-isopropyl-9,10a-dimethyl-6-methylenedodecahydro-1H-cyclopenta[ }\left[4^{\prime}, 5^{\prime}\right] \text { cycloocta[1',2':1,5] } \\
\text { cyclopenta[1,2-b]oxiren-4-ol }\end{array}$ & $\mathrm{C}_{20} \mathrm{H}_{32} \mathrm{O}_{2}$ \\
\hline 24 & 39.903 & 1912 & 1,2-Benzenedicarboxylic acid, butyl octyl ester & $\mathrm{C}_{20} \mathrm{H}_{30} \mathrm{O}_{4}$ \\
\hline 25 & 41.687 & 1951 & n-Hexadecanoic acid & $\mathrm{C}_{16} \mathrm{H}_{32} \mathrm{O}_{2}$ \\
\hline 26 & 49.181 & 2119 & 7-Hexadecenal, (Z)- & $\mathrm{C}_{16} \mathrm{H}_{30} \mathrm{O}$ \\
\hline 27 & 50.098 & 2139 & 9-Octadecenamide, (Z)- & $\mathrm{C}_{18} \mathrm{H}_{35} \mathrm{NO}$ \\
\hline 28 & 50.483 & 2148 & Octadecanoic acid & $\mathrm{C}_{18} \mathrm{H}_{36} \mathrm{O}_{2}$ \\
\hline 29 & 59.601 & 2362 & 2-Methyloctadecan-7,8-diol & $\mathrm{C}_{19} \mathrm{H}_{40} \mathrm{O}_{2}$ \\
\hline 30 & 65.148 & 2499 & 1,2-Benzenedicarboxylic acid, diisooctyl ester & $\mathrm{C}_{24} \mathrm{H}_{38} \mathrm{O}_{4}$ \\
\hline 31 & 73.761 & 2726 & 13-Docosenamide, $(\mathrm{Z})$ - & $\mathrm{C}_{22} \mathrm{H}_{43} \mathrm{NO}$ \\
\hline 32 & 77.825 & 2840 & 3-Phenyl-2-ethoxypropylphthalimide & $\mathrm{C}_{19} \mathrm{H}_{19} \mathrm{NO}_{3}$ \\
\hline 33 & 83.874 & 3017 & 9,10-Secocholesta-5,7,10(19)-triene-3,24,25-triol, (3.beta.,5Z,7E)- & $\mathrm{C}_{27} \mathrm{H}_{44} \mathrm{O}_{3}$ \\
\hline 34 & 89.708 & 3197 & Heptanoic acid, docosyl ester & $\mathrm{C}_{29} \mathrm{H}_{58} \mathrm{O}_{2}$ \\
\hline 35 & 92.123 & 3265 & Isophthalic acid, allyl pentadecyl ester & $\mathrm{C}_{26} \mathrm{H}_{40} \mathrm{O}_{4}$ \\
\hline 36 & 102.267 & 3561 & Benzenepropanoic acid, 3,5-bis(1,1-dimethylethyl)-4-hydroxy-, octadecyl ester & $\mathrm{C}_{35} \mathrm{H}_{62} \mathrm{O}_{3}$ \\
\hline
\end{tabular}

Note: The bold and italic fonts are used to refer to exclusive compounds. RT: Retention time. RI: Reflex index.

Table 2: List of the hydrophilic volatile compounds identified from the stem of the Ilex cornuta Lindl. \& Paxton.

\begin{tabular}{|c|c|c|c|c|}
\hline No & RT & RI & Compound \\
\hline 1 & 13.608 & 1198 & Molecular \\
\hline 2 & 19.258 & 1363 & 2-Furancarboxaldehyde, 5-(hydroxymethyl)- & $\mathrm{C}_{6} \mathrm{H}_{6} \mathrm{O}_{3}$ \\
\hline 3 & 21.565 & 1433 & 4-Hydroxy-2-methoxybenaldehyde & $\mathrm{C}_{8} \mathrm{H}_{8} \mathrm{O}_{3}$ \\
\hline 4 & 23.85 & 1504 & Cyclopentanemethanol,.alpha.-(1-methylethyl)-2-nitro-, [1.alpha. $\left(\mathrm{S}^{*}\right), 2$. alpha. $]$ & $\mathrm{C}_{9} \mathrm{H}_{17} \mathrm{NO}_{3}$ \\
\hline 5 & 24.743 & 1533 & $\mathrm{C}_{12} \mathrm{H}_{20} \mathrm{O}$ & 4,8-Decadienal, 5,9-dimethyl- \\
\hline
\end{tabular}


Luosheng Huang (2021) Chemical Composition Similarity Relationships among the Various Organs of the Ilex Cornuta Lindl. \& Paxton Based on the Analysis of Hydrophilic Volatile Compounds

\begin{tabular}{|c|c|c|c|c|}
\hline 6 & 25.469 & 1556 & Dodecanoic acid & $\mathrm{C}_{12} \mathrm{H}_{24} \mathrm{O}_{2}$ \\
\hline 7 & 25.681 & 1563 & 1-Cyclohexene-1-methanol, alpha.,2,6,6-tetramethyl- & $\mathrm{C}_{11} \mathrm{H}_{20} \mathrm{O}$ \\
\hline 8 & 26.105 & 1577 & Pentanoic acid, 2,2,4-trimethyl-3-carboxyisopropyl, isobutyl ester & $\mathrm{C}_{16} \mathrm{H}_{30} \mathrm{O}_{4}$ \\
\hline 9 & 26.245 & 1581 & Phenol, 3,4,5-trimethoxy- & $\mathrm{C}_{9} \mathrm{H}_{12} \mathrm{O}_{4}$ \\
\hline 10 & 26.495 & 1589 & 2-Methyl-4-(2,6,6-trimethylcyclohex-1-enyl)-but-2-en-1-ol & $\mathrm{C}_{14} \mathrm{H}_{24} \mathrm{O}$ \\
\hline 11 & 27.127 & 1608 & Benzaldehyde, 4-hydroxy-3,5-dimethoxy- & $\mathrm{C}_{9} \mathrm{H}_{10} \mathrm{O}_{4}$ \\
\hline 12 & 27.37 & 1614 & Ethanone, 1-[2-(5-hydroxy-1,1-dimethylhexyl)-3-methyl-2-cyclopropen-1-yl]- & $\mathrm{C}_{14} \mathrm{H}_{24} \mathrm{O}_{2}$ \\
\hline 13 & 27.88 & 1628 & Thieno[3,2-c]-pyridin-4(5H)-one & $\mathrm{C}_{7} \mathrm{H}_{5} \mathrm{NOS}$ \\
\hline 14 & 28.27 & 1638 & Spiro-[4.5]-decan-7-one, 1,8-dimethyl-8,9-epoxy-4-isopropyl- & $\mathrm{C}_{15} \mathrm{H}_{24} \mathrm{O}_{2}$ \\
\hline 15 & 28.685 & 1649 & 2-Bromo dodecane & $\mathrm{C}_{12} \mathrm{H}_{25} \mathrm{Br}$ \\
\hline 16 & 29.172 & 1662 & Ethanol, 2-(octadecyloxy)- & $\mathrm{C}_{20} \mathrm{H}_{42} \mathrm{O}_{2}$ \\
\hline 17 & 29.971 & 1683 & 1-(2-Hydroxy-4,5-dimethoxy-phenyl)-ethanone & $\mathrm{C}_{10} \mathrm{H}_{12} \mathrm{O}_{4}$ \\
\hline 18 & 30.271 & 1691 & 2-Propenal, 3-(4-hydroxy-3-methoxyphenyl)- & $\mathrm{C}_{10} \mathrm{H}_{10} \mathrm{O}_{3}$ \\
\hline 19 & 30.399 & 1694 & Butanol, 1-[2,2,3,3-tetramethyl-1-(3-methyl-1-penynyl)-cyclopropyl]- & $\mathrm{C}_{17} \mathrm{H}_{30} \mathrm{O}$ \\
\hline 20 & 30.641 & 1700 & Heptadecane & $\mathrm{C}_{17} \mathrm{H}_{36}$ \\
\hline 21 & 31.037 & 1710 & Hexadecane, 2,6,10,14-tetramethyl- & $\mathrm{C}_{20} \mathrm{H}_{42}$ \\
\hline 22 & 31.345 & 1717 & 4a-Dichloromethyl-4,4a,5,6,7,8-hexahydro-3H-naphthalen-2-one & $\mathrm{C}_{11} \mathrm{H}_{14} \mathrm{Cl}_{2} \mathrm{O}$ \\
\hline 23 & 31.75 & 1726 & Adamantane, 1-thiocyanatomethyl- & $\mathrm{C}_{12} \mathrm{H}_{17} \mathrm{NS}$ \\
\hline 24 & 32.052 & 1733 & 9-(3,3-Dimethyloxiran-2-yl)-2,7-dimethylnona-2,6-dien-1-ol & $\mathrm{C}_{15} \mathrm{H}_{26} \mathrm{O}_{2}$ \\
\hline 25 & 32.512 & 1744 & 1-Decanol, 2-hexyl- & $\mathrm{C}_{16} \mathrm{H}_{34} \mathrm{O}$ \\
\hline 26 & 32.788 & 1750 & Cyclopropane, 1-(1-hydroxy-1-heptyl)-2-methylene-3-pentyl- & $\mathrm{C}_{16} \mathrm{H}_{30} \mathrm{O}$ \\
\hline 27 & 33.683 & 1771 & 3-Isobutyryl-6-isopropyl-2,3-dihydropyran-2,4-dione & $\mathrm{C}_{12} \mathrm{H}_{16} \mathrm{O}_{4}$ \\
\hline 28 & 34.92 & 1800 & Heneicosane & $\mathrm{C}_{21} \mathrm{H}_{44}$ \\
\hline 29 & 35.489 & 1813 & Heptadecane, 2,6,10,15-tetramethyl- & $\mathrm{C}_{21} \mathrm{H}_{44}$ \\
\hline 30 & 35.86 & 1821 & 1,2-Benzenedicarboxylic acid, bis(2-methylpropyl) ester & $\mathrm{C}_{16} \mathrm{H}_{22} \mathrm{O}_{4}$ \\
\hline 31 & 37.316 & 1854 & 1-Hexadecanol & $\mathrm{C}_{16} \mathrm{H}_{34} \mathrm{O}$ \\
\hline 32 & 37.898 & 1867 & $\begin{array}{l}\text { 2a-isopropyl-9,10a-dimethyl-6-methylenedodecahydro-1H-cyclopenta[4',5']-cycloocta[1',2':1,5]-cyclopenta- } \\
\text { [1,2-b]oxiren-4-ol }\end{array}$ & $\mathrm{C}_{20} \mathrm{H}_{32} \mathrm{O}_{2}$ \\
\hline 33 & 39.907 & 1912 & 1,2-Benzenedicarboxylic acid, butyl 8-methylnonyl ester & $\mathrm{C}_{22} \mathrm{H}_{34} \mathrm{O}_{4}$ \\
\hline 34 & 41.693 & 1951 & $\mathrm{n}$-Hexadecanoic acid & $\mathrm{C}_{16} \mathrm{H}_{32} \mathrm{O}_{2}$ \\
\hline 35 & 43.899 & 2000 & Eicosane & $\mathrm{C}_{20} \mathrm{H}_{42}$ \\
\hline 36 & 49.179 & 2119 & 12-Methyl-E,E-2,13-octadecadien-1-ol & $\mathrm{C}_{19} \mathrm{H}_{36} \mathrm{O}$ \\
\hline 37 & 50.464 & 2148 & Octadecanoic acid & $\mathrm{C}_{18} \mathrm{H}_{36} \mathrm{O}_{2}$ \\
\hline 38 & 59.603 & 2362 & 2-Methyloctadecan-7,8-diol & $\mathrm{C}_{19} \mathrm{H}_{40} \mathrm{O}_{2}$ \\
\hline 39 & 65.152 & 2499 & 1,2-Benzenedicarboxylic acid, diisooctyl ester & $\mathrm{C}_{24} \mathrm{H}_{38} \mathrm{O}_{4}$ \\
\hline 40 & 73.757 & 2726 & 13-Docosenamide, (Z)- & $\mathrm{C}_{22} \mathrm{H}_{43} \mathrm{NO}$ \\
\hline 41 & 83.861 & 3016 & Ethyl iso-allocholate & $\mathrm{C}_{26} \mathrm{H}_{44} \mathrm{O}_{5}$ \\
\hline 42 & 89.711 & 3197 & Heptanoic acid, docosyl ester & $\mathrm{C}_{29} \mathrm{H}_{58} \mathrm{O}_{2}$ \\
\hline 43 & 92.16 & 3266 & Isophthalic acid, allyl pentadecyl ester & $\mathrm{C}_{26} \mathrm{H}_{40} \mathrm{O}_{4}$ \\
\hline 44 & 92.66 & 3280 & 17-(1,5-Dimethylhexyl)-10,13-dimethyl-3-styrylhexadecahydrocyclopenta[a]phenanthren-2-one & $\mathrm{C}_{35} \mathrm{H}_{52} \mathrm{O}$ \\
\hline 45 & 94.571 & 3331 & 4-Norlanosta-17(20),24-diene-11,16-diol-21-oic acid, 3-oxo-16,21-lactone & $\mathrm{C}_{29} \mathrm{H}_{42} \mathrm{O}_{4}$ \\
\hline 46 & 102.263 & 3561 & Benzenepropanoic acid, 3,5-bis(1,1-dimethylethyl)-4-hydroxy-, octadecyl ester & $\mathrm{C}_{35} \mathrm{H}_{62} \mathrm{O}_{3}$ \\
\hline
\end{tabular}

Note: The bold and italic fonts are used to refer to exclusive compounds. RT: Retention time. RI: Reflex index. 
Luosheng Huang (2021) Chemical Composition Similarity Relationships among the Various Organs of the Ilex Cornuta Lindl. \& Paxton Based on the Analysis of Hydrophilic Volatile Compounds

Table 3: List of the hydrophilic volatile component identified from the stem skin of the Ilex cornuta Lindl. \& Paxton.

\begin{tabular}{|c|c|c|c|c|}
\hline No & RT & RI & Compound & Molecular \\
\hline 1 & 12.765 & 1173 & Octanoic Acid & $\mathrm{C}_{8} \mathrm{H}_{16} \mathrm{O}_{2}$ \\
\hline 2 & 13.818 & 1204 & 2-Furancarboxaldehyde, 5-(hydroxymethyl)- & $\mathrm{C}_{6} \mathrm{H}_{6} \mathrm{O}_{3}$ \\
\hline 3 & 19.283 & 1364 & Benzaldehyde, 3-hydroxy-4-methoxy- & $\mathrm{C}_{8} \mathrm{H}_{8} \mathrm{O}_{3}$ \\
\hline 4 & 21.526 & 1432 & 2H-Pyran-2-one, 5,6-dihydro-6-pentyl- & $\mathrm{C}_{10} \mathrm{H}_{16} \mathrm{O}_{2}$ \\
\hline 5 & 23.372 & 1489 & 4,6-di-tert-Butyl-m-cresol & $\mathrm{C}_{15} \mathrm{H}_{24} \mathrm{O}$ \\
\hline 6 & 23.599 & 1496 & 12-Oxa-[tetracyclo[5.2.1.1(2,6).1(8,11)]]dodecan-10-ol, 3-acetoxy- & $\mathrm{C}_{13} \mathrm{H}_{18} \mathrm{O}_{4}$ \\
\hline 7 & 23.856 & 1504 & 2,6-Dimethoxybenzoquinone & $\mathrm{C}_{8} \mathrm{H}_{8} \mathrm{O}_{4}$ \\
\hline 8 & 25.171 & 1547 & 1H-Benzocyclohepten-7-ol, 2,3,4,4a,5,6,7,8-octahydro-1,1,4a,7-tetramethyl-, cis- & $\mathrm{C}_{15} \mathrm{H}_{26} \mathrm{O}$ \\
\hline 9 & 25.317 & 1551 & 2(5H)-Furanone, 4-methyl-5,5-bis(2-methyl-2-propenyl)- & $\mathrm{C}_{13} \mathrm{H}_{18} \mathrm{O}_{2}$ \\
\hline 10 & 25.462 & 1556 & Dodecanoic acid & $\mathrm{C}_{12} \mathrm{H}_{24} \mathrm{O}_{2}$ \\
\hline 11 & 25.694 & 1564 & 2-Oxabicyclo[3.3.0]oct-7-en-3-one, 7-(1-hydroxypentyl)- & $\mathrm{C}_{12} \mathrm{H}_{18} \mathrm{O}_{3}$ \\
\hline 12 & 25.922 & 1571 & Dodecane, 2,6,10-trimethyl- & $\mathrm{C}_{15} \mathrm{H}_{32}$ \\
\hline 13 & 26.114 & 1577 & Pentanoic acid, 2,2,4-trimethyl-3-carboxyisopropyl, isobutyl ester & $\mathrm{C}_{16} \mathrm{H}_{30} \mathrm{O}_{4}$ \\
\hline 14 & 26.335 & 1584 & 3-Butyl-4-nitro-pent-4-enoic acid, methyl ester & $\mathrm{C}_{10} \mathrm{H}_{1} \mathrm{NO}_{4}$ \\
\hline 15 & 26.514 & 1590 & 2-Dodecen-1-yl(-)succinic anhydride & $\mathrm{C}_{16} \mathrm{H}_{26} \mathrm{O}_{3}$ \\
\hline 16 & 26.838 & 1600 & Heptadecane & $\mathrm{C}_{17} \mathrm{H}_{36}$ \\
\hline 17 & 27.227 & 1611 & Benzaldehyde, 4-hydroxy-3,5-dimethoxy- & $\mathrm{C}_{9} \mathrm{H}_{10} \mathrm{O}_{4}$ \\
\hline 18 & 27.929 & 1629 & 2,6,10,10-Tetramethyl-1-oxaspiro-[4.5]decan-6-ol & $\mathrm{C}_{13} \mathrm{H}_{24} \mathrm{O}_{2}$ \\
\hline 19 & 28.288 & 1638 & 4-Isobenzofuranol, octahydro-3a,7a-dimethyl-, (3a.alpha.,4.beta.,7a.alpha.)-(.+-.)- & $\mathrm{C}_{10} \mathrm{H}_{18} \mathrm{O}_{2}$ \\
\hline 20 & 29.187 & 1662 & Ethanol, 2-(hexadecyloxy)- & $\mathrm{C}_{18} \mathrm{H}_{38} \mathrm{O}_{2}$ \\
\hline 21 & 29.827 & 1679 & 2-Cyclohexen-1-one, 3-(3-hydroxybutyl)-2,4,4-trimethyl- & $\mathrm{C}_{13} \mathrm{H}_{22} \mathrm{O}_{2}$ \\
\hline 22 & 29.956 & 1682 & Cyclopentanone, 2-(1-adamantyl)- & $\mathrm{C}_{15} \mathrm{H}_{22} \mathrm{O}$ \\
\hline 23 & 30.308 & 1692 & alpha. Isomethyl ionone & $\mathrm{C}_{14} \mathrm{H}_{22} \mathrm{O}$ \\
\hline 24 & 30.649 & 1700 & 2-Bromotetradecane & $\mathrm{C}_{14} \mathrm{H}_{29} \mathrm{Br}$ \\
\hline 25 & 31.047 & 1710 & Hexadecane, 2,6,10,14-tetramethyl- & $\mathrm{C}_{20} \mathrm{H}_{42}$ \\
\hline 26 & 31.774 & 1727 & Adamantane, 1-thiocyanatomethyl- & $\mathrm{C}_{12} \mathrm{H}_{17} \mathrm{NS}$ \\
\hline 27 & 32.083 & 1734 & E,E-6,8-Tridecadien-2-ol, acetate & $\mathrm{C}_{15} \mathrm{H}_{26} \mathrm{O}_{2}$ \\
\hline 28 & 32.522 & 1744 & 1-Decanol, 2-hexyl- & $\mathrm{C}_{16} \mathrm{H}_{34} \mathrm{O}$ \\
\hline 29 & 32.801 & 1751 & 7-Methyl-Z-tetradecen-1-ol acetate & $\mathrm{C}_{17} \mathrm{H}_{32} \mathrm{O}_{2}$ \\
\hline 30 & 33.682 & 1771 & 7-Bromo-3a,6,6-trimethyl-hexahydro-benzofuran-2(3H)-one & $\mathrm{C}_{11} \mathrm{H}_{17} \mathrm{BrO}_{2}$ \\
\hline 31 & 35.475 & 1813 & Heptadecane, 2,6,10,15-tetramethyl- & $\mathrm{C}_{21} \mathrm{H}_{44}$ \\
\hline 32 & 35.876 & 1822 & 1,2-Benzenedicarboxylic acid, bis(2-methylpropyl) ester & $\mathrm{C}_{16} \mathrm{H}_{22} \mathrm{O}_{4}$ \\
\hline 33 & 37.903 & 1867 & Dodecane, 1,2-dibromo- & $\mathrm{C}_{12} \mathrm{H}_{24} \mathrm{Br}_{2}$ \\
\hline 34 & 39.916 & 1912 & Dibutyl phthalate & $\mathrm{C}_{16} \mathrm{H}_{22} \mathrm{O}_{4}$ \\
\hline 35 & 41.653 & 1951 & n-Hexadecanoic acid & $\mathrm{C}_{16} \mathrm{H}_{32} \mathrm{O}_{2}$ \\
\hline 36 & 65.178 & 2500 & 1,2-Benzenedicarboxylic acid, diisooctyl ester & $\mathrm{C}_{24} \mathrm{H}_{38} \mathrm{O}_{4}$ \\
\hline 37 & 73.760 & 2726 & 13-Docosenamide, $(Z)$ - & $\mathrm{C}_{22} \mathrm{H}_{43} \mathrm{NO}$ \\
\hline 38 & 93.411 & 3301 & 1,2-Benzenedicarboxylic acid, diundecyl ester & $\mathrm{C}_{30} \mathrm{H}_{50} \mathrm{O}_{4}$ \\
\hline 39 & 93.650 & 3307 & Isophthalic acid, allyl pentadecyl ester & $\mathrm{C}_{26} \mathrm{H}_{40} \mathrm{O}_{4}$ \\
\hline 40 & 100.522 & 3505 & 9-Octadecenoic acid (Z)-, phenylmethyl ester & $\mathrm{C}_{25} \mathrm{H}_{40} \mathrm{O}_{2}$ \\
\hline 41 & 101.150 & 3525 & 2,6-Lutidine 3,5-dichloro-4-dodecylthio- & $\mathrm{C}_{19} \mathrm{H}_{31} \mathrm{Cl}_{2} \mathrm{NS}$ \\
\hline 42 & 102.301 & 3562 & Benzenepropanoic acid, 3,5-bis(1,1-dimethylethyl)-4-hydroxy-, octadecyl ester & $\mathrm{C}_{35} \mathrm{H}_{62} \mathrm{O}_{3}$ \\
\hline
\end{tabular}

Note: The bold and italic fonts are used to refer to exclusive compounds. RT: Retention time. RI: Reflex index. 
Luosheng Huang (2021) Chemical Composition Similarity Relationships among the Various Organs of the Ilex Cornuta Lindl. \& Paxton Based on the Analysis of Hydrophilic Volatile Compounds

Table 4: List of the hydrophilic volatile component of the leaf of the Ilex cornuta Lindl. \& Paxton.

\begin{tabular}{|c|c|c|c|c|}
\hline No & RT & RI & Compound & Molecular \\
\hline 1 & 13.543 & 1196 & 2-Furancarboxaldehyde, 5-(hydroxymethyl)- & $\mathrm{C}_{6} \mathrm{H}_{6} \mathrm{O}_{3}$ \\
\hline 2 & 14.110 & 1212 & 2-Furancarboxaldehyde, 6-(hydroxymethyl)- & $\mathrm{C}_{6} \mathrm{H}_{6} \mathrm{O}_{4}$ \\
\hline 3 & 14.318 & 1218 & 2-Furancarboxaldehyde, 7-(hydroxymethyl)- & $\mathrm{C}_{6} \mathrm{H}_{6} \mathrm{O}_{5}$ \\
\hline 4 & 25.089 & 1544 & Bicyclo[3.2.0]heptan-6-one, 2-acetyl-3,3-dimethyl-7-(1-methylethyl)- & $\mathrm{C}_{14} \mathrm{H}_{22} \mathrm{O}_{2}$ \\
\hline 5 & 25.453 & 1556 & Dodecanoic acid & $\mathrm{C}_{12} \mathrm{H}_{24} \mathrm{O}_{2}$ \\
\hline 6 & 25.692 & 1564 & trans-Z-.alpha.-Bisabolene epoxide & $\mathrm{C}_{15} \mathrm{H}_{24} \mathrm{O}$ \\
\hline 7 & 26.117 & 1577 & 4,6,10,10-Tetramethyl-5-oxatricyclo[4.4.0.0(1,4)]dec-2-en-7-ol & $\mathrm{C}_{13} \mathrm{H}_{20} \mathrm{O}_{2}$ \\
\hline 8 & 26.493 & 1589 & 7-Heptadecene, 1-chloro- & $\mathrm{C}_{17} \mathrm{H}_{33} \mathrm{Cl}$ \\
\hline 9 & 26.831 & 1600 & Hexadecane & $\mathrm{C}_{16} \mathrm{H}_{34}$ \\
\hline 10 & 28.088 & 1633 & 3-Pyridinecarboxylic acid, 1,6-dihydro-4-hydroxy-2-methyl-6-oxo-, ethyl ester & $\mathrm{C}_{9} \mathrm{H}_{11} \mathrm{NO}_{4}$ \\
\hline 11 & 30.644 & 1700 & Heptadecane & $\mathrm{C}_{17} \mathrm{H}_{36}$ \\
\hline 12 & 31.038 & 1710 & Hexadecane, 2,6,11,15-tetramethyl- & $\mathrm{C}_{20} \mathrm{H}_{42}$ \\
\hline 13 & 32.440 & 1742 & 2-Cyclohexen-1-one, 4-hydroxy-3,5,6-trimethyl-4-(3-oxo-1-butenyl)- & $\mathrm{C}_{13} \mathrm{H}_{18} \mathrm{O}_{3}$ \\
\hline 14 & 32.801 & 1751 & 7-Methyl-Z-tetradecen-1-ol acetate & $\mathrm{C}_{17} \mathrm{H}_{32} \mathrm{O}_{2}$ \\
\hline 15 & 34.479 & 1790 & Pentadecyl trifluoroacetate & $\mathrm{C}_{17} \mathrm{H}_{31} \mathrm{~F}_{3} \mathrm{O}_{2}$ \\
\hline 16 & 34.925 & 1800 & Heptadecane, 2,6,10,15-tetramethyl- & $\mathrm{C}_{21} \mathrm{H}_{44}$ \\
\hline 17 & 35.476 & 1813 & Nonadecane & $\mathrm{C}_{19} \mathrm{H}_{40}$ \\
\hline 18 & 35.872 & 1822 & 1,2-Benzenedicarboxylic acid, bis(2-methylpropyl) ester & $\mathrm{C}_{16} \mathrm{H}_{22} \mathrm{O}_{4}$ \\
\hline 19 & 41.601 & 1949 & n-Hexadecanoic acid & $\mathrm{C}_{16} \mathrm{H}_{32} \mathrm{O}_{2}$ \\
\hline 20 & 43.458 & 1991 & 1-Nonadecene & $\mathrm{C}_{19} \mathrm{H}_{38}$ \\
\hline 21 & 59.608 & 2362 & 2-Methyloctadecan-7,8-diol & $\mathrm{C}_{19} \mathrm{H}_{40} \mathrm{O}_{2}$ \\
\hline 22 & 73.760 & 2726 & 13-Docosenamide, $(\mathrm{Z})$ - & $\mathrm{C}_{22} \mathrm{H}_{43} \mathrm{NO}$ \\
\hline 23 & 92.239 & 3268 & Isophthalic acid, allyl pentadecyl ester & $\mathrm{C}_{26} \mathrm{H}_{40} \mathrm{O}_{4}$ \\
\hline 24 & 93.235 & 3296 & 1,2-Benzenedicarboxylic acid, 2-butoxyethyl butyl ester & $\mathrm{C}_{18} \mathrm{H}_{26} \mathrm{O}_{5}$ \\
\hline 25 & 94.006 & 3317 & Phthalic acid, propyl octadecyl ester & $\mathrm{C}_{29} \mathrm{H}_{48} \mathrm{O}_{4}$ \\
\hline
\end{tabular}

Table 5: List of the hydrophilic volatile component identified from the flower of the Ilex cornuta Lindl. \& Paxton.

\begin{tabular}{|c|c|c|c|c|}
\hline No & RT & RI & Compound & Molecular \\
\hline 1 & 9.359 & 1070 & 2,2-Dimethyl-3-vinyl-bicyclo[2.2.1]heptane & $\mathrm{C}_{11} \mathrm{H}_{18}$ \\
\hline 2 & 9.987 & 1091 & Cyclohex-3-enecarboxaldehyde, 2,4,6-trimethyl-, oxime & $\mathrm{C}_{10} \mathrm{H}_{1} \mathrm{NO}$ \\
\hline 3 & 12.197 & 1156 & Phenol, 3-ethyl- & $\mathrm{C}_{8} \mathrm{H}_{10} \mathrm{O}$ \\
\hline 4 & 12.649 & 1170 & Benzoic acid & $\mathrm{C}_{7} \mathrm{H}_{6} \mathrm{O}_{2}$ \\
\hline 5 & 12.797 & 1174 & Glucosamine, N-acetyl-N-benzoyl- & $\mathrm{C}_{15} \mathrm{H}_{19} \mathrm{NO}_{7}$ \\
\hline 6 & 13.333 & 1190 & Benzothiazole & $\mathrm{C}_{7} \mathrm{H}_{5} \mathrm{NS}$ \\
\hline 7 & 15.613 & 1256 & Phenol, 2,3,5-trimethyl- & $\mathrm{C}_{9} \mathrm{H}_{12} \mathrm{O}$ \\
\hline 8 & 16.214 & 1273 & 5H-Inden-5-one, 1,2,3,6,7,7a-hexahydro- & $\mathrm{C}_{9} \mathrm{H}_{12} \mathrm{O}$ \\
\hline 9 & 16.640 & 1286 & Hydroquinone & $\mathrm{C}_{6} \mathrm{H}_{6} \mathrm{O}_{2}$ \\
\hline 10 & 17.145 & 1300 & Cyclohexanol, 1-methyl-4-(1-methylethylidene)- & $\mathrm{C}_{10} \mathrm{H}_{18} \mathrm{O}$ \\
\hline 11 & 17.280 & 1304 & Cyclohexanol, 2-methyl-5-(1-methylethenyl)-, (1.alpha.,2.beta.,5.alpha.)- & $\mathrm{C}_{10} \mathrm{H}_{18} \mathrm{O}$ \\
\hline 12 & 17.772 & 1319 & 2,7-Octadiene-1,6-diol, 2,6-dimethyl- & $\mathrm{C}_{10} \mathrm{H}_{18} \mathrm{O}_{2}$ \\
\hline 13 & 18.160 & 1330 & trans-Z-.alpha.-Bisabolene epoxide & $\mathrm{C}_{15} \mathrm{H}_{24} \mathrm{O}$ \\
\hline 14 & 18.430 & 1338 & $(3 S, 4 R, 5 R, 6 R)-4,5$-Bis(hydroxymethyl)-3,6-dimethylcyclohexene & $\mathrm{C}_{10} \mathrm{H}_{18} \mathrm{O}_{2}$ \\
\hline 15 & 19.298 & 1364 & 4-Hydroxy-2-methoxybenaldehyde & $\mathrm{C}_{8} \mathrm{H}_{8} \mathrm{O}_{3}$ \\
\hline 16 & 19.508 & 1370 & 2-Cyclopenten-1-one, 4-hydroxy-3-methyl-2-(2-propenyl)- & $\mathrm{C}_{9} \mathrm{H}_{12} \mathrm{O}_{2}$ \\
\hline 17 & 21.040 & 1417 & Phenol, 2-pentyl- & $\mathrm{C}_{11} \mathrm{H}_{16} \mathrm{O}$ \\
\hline 18 & 21.311 & 1425 & 2-Propen-1-ol, 2-methyl-3-(2,6,6-trimethyl-2-cyclohexen-1-yl)-, (E)- & $\mathrm{C}_{13} \mathrm{H}_{22} \mathrm{O}$ \\
\hline 19 & 21.602 & 1434 & 3-(2-Hydroxy-cyclopentylidene)-2-methyl-propionic acid & $\mathrm{C}_{9} \mathrm{H}_{14} \mathrm{O}_{3}$ \\
\hline
\end{tabular}


Luosheng Huang (2021) Chemical Composition Similarity Relationships among the Various Organs of the Ilex Cornuta Lindl. \& Paxton Based on the Analysis of Hydrophilic Volatile Compounds

\begin{tabular}{|c|c|c|c|c|}
\hline 20 & 21.838 & 1442 & 5-Benzofuranacetic acid, 6-ethenyl-2,4,5,6,7,7a-hexahydro-3,6-dimethyl- $\alpha$-methylene-2-oxo-, methyl ester & $\mathrm{C}_{16} \mathrm{H}_{20} \mathrm{O}_{4}$ \\
\hline 21 & 23.259 & 1486 & 8-Methylenecyclooctene-3,4-diol & $\mathrm{C}_{9} \mathrm{H}_{14} \mathrm{O}_{2}$ \\
\hline 22 & 23.514 & 1494 & 1-(3,6,6-Trimethyl-1,6,7,7a-tetrahydrocyclopenta[c]pyran-1-yl)ethanone & $\mathrm{C}_{13} \mathrm{H}_{18} \mathrm{O}_{2}$ \\
\hline 23 & 24.011 & 1509 & 1-Acetamido-1,2-dihydro-2-oxopyridine & $\mathrm{C}_{7} \mathrm{H}_{8} \mathrm{~N}_{2} \mathrm{O}_{2}$ \\
\hline 24 & 24.675 & 1531 & cis-Z-alpha.-Bisabolene epoxide & $\mathrm{C}_{15} \mathrm{H}_{24} \mathrm{O}$ \\
\hline 25 & 24.767 & 1534 & Cyclopentan-1-al, 4-isopropylidene-2-methyl- & $\mathrm{C}_{10} \mathrm{H}_{16} \mathrm{O}$ \\
\hline 26 & 25.085 & 1544 & Ethanone, 1-(1a,2,3,5,6a,6b-hexahydro-3,3,6a-trimethyloxireno[g]benzofuran-5-yl)- & $\mathrm{C}_{13} \mathrm{H}_{18} \mathrm{O}_{3}$ \\
\hline 27 & 25.514 & 1558 & Dodecanoic acid & $\mathrm{C}_{12} \mathrm{H}_{24} \mathrm{O}_{2}$ \\
\hline 28 & 25.685 & 1563 & Bicyclo[3.3.1]nonan-9-one, 1,2,4-trimethyl-3-nitro-, (2-endo,3-exo,4-exo)-(.+-.)- & $\mathrm{C}_{12} \mathrm{H}_{19} \mathrm{NO}_{3}$ \\
\hline 29 & 25.899 & 1570 & 2-Cyclohexen-1-one, 3-(3-hydroxybutyl)-2,4,4-trimethyl- & $\mathrm{C}_{13} \mathrm{H}_{22} \mathrm{O}_{2}$ \\
\hline 30 & 26.127 & 1578 & Ledol & $\mathrm{C}_{15} \mathrm{H}_{26} \mathrm{O}$ \\
\hline 31 & 26.498 & 1590 & 1-Hexadecanol & $\mathrm{C}_{16} \mathrm{H}_{34} \mathrm{O}$ \\
\hline 32 & 26.840 & 1600 & Hexadecane & $\mathrm{C}_{16} \mathrm{H}_{34}$ \\
\hline 33 & 27.155 & 1609 & Spiro[androst-5-ene-17,1'-cyclobutan]-2'-one, 3-hydroxy-, (3.beta.,17.beta.)- & $\mathrm{C}_{22} \mathrm{H}_{32} \mathrm{O}_{2}$ \\
\hline 34 & 27.486 & 1617 & Bicyclo[3.1.0]hexane-6-methanol, 2-hydroxy-1,4,4-trimethyl- & $\mathrm{C}_{10} \mathrm{H}_{18} \mathrm{O}_{2}$ \\
\hline 35 & 28.099 & 1634 & 3-Pyridinecarboxylic acid, 1,6-dihydro-4-hydroxy-2-methyl-6-oxo-, ethyl ester & $\mathrm{C}_{9} \mathrm{H}_{11} \mathrm{NO}_{4}$ \\
\hline 36 & 28.615 & 1647 & Bromoacetic acid, dodecyl ester & $\mathrm{C}_{14} \mathrm{H}_{27} \mathrm{BrO}_{2}$ \\
\hline 37 & 28.684 & 1649 & Chloroacetic acid, 4-tetradecyl ester & $\mathrm{C}_{16} \mathrm{H}_{31} \mathrm{ClO}_{2}$ \\
\hline 38 & 29.178 & 1662 & 2-Dodecen-1-yl(-)succinic anhydride & $\mathrm{C}_{16} \mathrm{H}_{26} \mathrm{O}_{3}$ \\
\hline 39 & 29.777 & 1678 & 2-Hydroxy-1,1,10-trimethyl-6,9-epidioxydecalin & $\mathrm{C}_{13} \mathrm{H}_{22} \mathrm{O}_{3}$ \\
\hline 40 & 29.951 & 1682 & 1-Cyclopropene-1-pentanol, alpha.,.epsilon.,.epsilon.,2-tetramethyl-3-(1-methylethenyl)- & $\mathrm{C}_{15} \mathrm{H}_{26} \mathrm{O}$ \\
\hline 41 & 30.651 & 1701 & 2-Bromotetradecane & $\mathrm{C}_{14} \mathrm{H}_{29} \mathrm{Br}$ \\
\hline 42 & 31.045 & 1710 & Tetradecane, 1-chloro- & $\mathrm{C}_{14} \mathrm{H}_{29} \mathrm{Cl}$ \\
\hline 43 & 31.355 & 1717 & 5.beta.,7.beta.H,10.alpha.-Eudesm-11-en-1.alpha.-ol & $\mathrm{C}_{15} \mathrm{H}_{26} \mathrm{O}$ \\
\hline 44 & 31.582 & 1722 & 7-Hexadecenal, (Z)- & $\mathrm{C}_{16} \mathrm{H}_{30} \mathrm{O}$ \\
\hline 45 & 31.771 & 1727 & Pentane-2,4-dione, 3-(1-adamantyl)- & $\mathrm{C}_{15} \mathrm{H}_{22} \mathrm{O}_{2}$ \\
\hline 46 & 32.092 & 1734 & Butanol, 1-[2,2,3,3-tetramethyl-1-(3-methyl-1-penynyl)-cyclopropyl]- & $\mathrm{C}_{17} \mathrm{H}_{30} \mathrm{O}$ \\
\hline 47 & 32.468 & 1743 & Pyrrolo[1,2-a]pyrazine-1,4-dione, hexahydro-3-(2-methylpropyl)- & $\mathrm{C}_{11} \mathrm{H}_{18} \mathrm{~N}_{2} \mathrm{O}_{2}$ \\
\hline 48 & 32.769 & 1750 & Tetradecanoic acid & $\mathrm{C}_{14} \mathrm{H}_{28} \mathrm{O}_{2}$ \\
\hline 49 & 33.645 & 1771 & 1-Decanol, 2-hexyl- & $\mathrm{C}_{16} \mathrm{H}_{34} \mathrm{O}$ \\
\hline 50 & 34.485 & 1790 & Pentadecyl trifluoroacetate & $\mathrm{C}_{17} \mathrm{H}_{31} \mathrm{~F}_{3} \mathrm{O}_{2}$ \\
\hline 51 & 34.932 & 1801 & Heptadecane, 2,6,10,15-tetramethyl- & $\mathrm{C}_{21} \mathrm{H}_{44}$ \\
\hline 52 & 35.479 & 1813 & 1-Octanol, 2-butyl- & $\mathrm{C}_{12} \mathrm{H}_{26} \mathrm{O}$ \\
\hline 53 & 35.873 & 1822 & 1,2-Benzenedicarboxylic acid, bis(2-methylpropyl) ester & $\mathrm{C}_{16} \mathrm{H}_{22} \mathrm{O}_{4}$ \\
\hline 54 & 36.928 & 1845 & 5,10-Diethoxy-2,3,7,8-tetrahydro-1H,6H-dipyrrolo $\left[1,2-a ; 1^{\prime}, 2^{\prime}\right.$-d]pyrazine & $\mathrm{C}_{14} \mathrm{H}_{22} \mathrm{~N}_{2} \mathrm{O}_{2}$ \\
\hline 55 & 37.910 & 1867 & 2-Hexadecene, 3,7,11,15-tetramethyl-, $\left[R-\left[R^{*}, R^{*}-(E)\right]\right]$ - & $\mathrm{C}_{20} \mathrm{H}_{40}$ \\
\hline 56 & 39.389 & 1900 & Nonadecane & $\mathrm{C}_{19} \mathrm{H}_{40}$ \\
\hline 57 & 41.675 & 1951 & $\mathrm{n}$-Hexadecanoic acid & $\mathrm{C}_{16} \mathrm{H}_{32} \mathrm{O}_{2}$ \\
\hline 58 & 43.480 & 1991 & 1-Nonadecene & $\mathrm{C}_{19} \mathrm{H}_{38}$ \\
\hline 59 & 46.996 & 2069 & 3-Chloropropionic acid, heptadecyl ester & $\mathrm{C}_{20} \mathrm{H}_{39} \mathrm{ClO}_{2}$ \\
\hline 60 & 48.984 & 2114 & 9,12-Octadecadienoic acid $(Z, Z)$ - & $\mathrm{C}_{18} \mathrm{H}_{32} \mathrm{O}_{2}$ \\
\hline 61 & 49.228 & 2120 & 9-Octadecenal, (Z)- & $\mathrm{C}_{18} \mathrm{H}_{34} \mathrm{O}$ \\
\hline 62 & 50.660 & 2152 & Ethyl iso-allocholate & $\mathrm{C}_{26} \mathrm{H}_{44} \mathrm{O}_{5}$ \\
\hline 63 & 52.394 & 2192 & 9-Tricosene, (Z)- & $\mathrm{C}_{23} \mathrm{H}_{46}$ \\
\hline 64 & 73.770 & 2727 & 13-Docosenamide, (Z)- & $\mathrm{C}_{22} \mathrm{H}_{43} \mathrm{NO}$ \\
\hline
\end{tabular}

Note: The bold and italic fonts are used to refer to exclusive compounds. RT: Retention time. RI: Reflex index. 
Luosheng Huang (2021) Chemical Composition Similarity Relationships among the Various Organs of the Ilex Cornuta Lindl. \& Paxton Based on the Analysis of Hydrophilic Volatile Compounds

Table 6: List of the hydrophilic volatile component identified from the fruit of the Ilex cornuta Lindl. \& Paxton.

\begin{tabular}{|c|c|c|c|c|}
\hline No & RT & RI & Compound & Molecular \\
\hline 1 & 9.133 & 1063 & Mequinol & $\mathrm{C}_{7} \mathrm{H}_{8} \mathrm{O}_{2}$ \\
\hline 2 & 9.303 & 1069 & Phenol, 4-methyl- & $\mathrm{C}_{7} \mathrm{H}_{8} \mathrm{O}$ \\
\hline 3 & 9.430 & 1073 & Hexane, 3-bromo- & $\mathrm{C}_{6} \mathrm{H}_{13} \mathrm{Br}$ \\
\hline 4 & 9.923 & 1089 & Phenylethyl Alcohol & $\mathrm{C}_{8} \mathrm{H}_{10} \mathrm{O}$ \\
\hline 5 & 10.510 & 1107 & 4-Acetylbutyric acid & $\mathrm{C}_{6} \mathrm{H}_{10} \mathrm{O}_{3}$ \\
\hline 6 & 12.643 & 1169 & Benzoic acid & $\mathrm{C}_{7} \mathrm{H}_{6} \mathrm{O}_{2}$ \\
\hline 7 & 13.559 & 1196 & 2-Furancarboxaldehyde, 5-(hydroxymethyl)- & $\mathrm{C}_{6} \mathrm{H}_{6} \mathrm{O}_{3}$ \\
\hline 8 & 15.378 & 1249 & 1,5-Cyclooctadien-4-one & $\mathrm{C}_{8} \mathrm{H}_{10} \mathrm{O}$ \\
\hline 9 & 17.652 & 1315 & Phenol, 2,6-dimethoxy- & $\mathrm{C}_{8} \mathrm{H}_{10} \mathrm{O}_{3}$ \\
\hline 10 & 19.180 & 1361 & Benzaldehyde, 3-hydroxy-4-methoxy- & $\mathrm{C}_{8} \mathrm{H}_{8} \mathrm{O}_{3}$ \\
\hline 11 & 21.852 & 1442 & 2-Ethoxyphenylacetonitrile & $\mathrm{C}_{10} \mathrm{H}_{11} \mathrm{NO}$ \\
\hline 12 & 22.103 & 1450 & Benzeneacetonitrile, 4-hydroxy- & $\mathrm{C}_{8} \mathrm{H}_{7} \mathrm{NO}$ \\
\hline 13 & 22.466 & 1461 & Coumarin, 8-methyl- & $\mathrm{C}_{10} \mathrm{H}_{8} \mathrm{O}_{2}$ \\
\hline 14 & 25.187 & 1547 & 1,4-Benzenediol, 2-(1,1-dimethylethyl)- & $\mathrm{C}_{10} \mathrm{H}_{14} \mathrm{O}_{2}$ \\
\hline 15 & 25.508 & 1558 & Dodecanoic acid & $\mathrm{C}_{12} \mathrm{H}_{24} \mathrm{O}_{2}$ \\
\hline 16 & 25.876 & 1569 & 3,5-Octadienoic acid, 7-hydroxy-2-methyl-, $\left[R^{*}, R^{*}-(E, E)\right]-$ & $\mathrm{C}_{9} \mathrm{H}_{14} \mathrm{O}_{3}$ \\
\hline 17 & 25.938 & 1571 & 2-Cyclopenten-1-one, 4-hydroxy-3-methyl-2-(2-propenyl)- & $\mathrm{C}_{9} \mathrm{H}_{12} \mathrm{O}_{2}$ \\
\hline 18 & 26.125 & 1577 & 1b,5,5,6a-Tetramethyl-octahydro-1-oxa-cyclopropa[a]inden-6-one & $\mathrm{C}_{13} \mathrm{H}_{20} \mathrm{O}_{2}$ \\
\hline 19 & 26.492 & 1589 & 4-Chloro-3-n-hexyltetrahydropyran & $\mathrm{C}_{11} \mathrm{H}_{21} \mathrm{ClO}$ \\
\hline 20 & 27.323 & 1613 & Ethanone, 1-[2-(5-hydroxy-1,1-dimethylhexyl)-3-methyl-2-cyclopropen-1-yl]- & $\mathrm{C}_{14} \mathrm{H}_{24} \mathrm{O}_{2}$ \\
\hline 21 & 30.643 & 1700 & Heptadecane & $\mathrm{C}_{17} \mathrm{H}_{36}$ \\
\hline 22 & 32.760 & 1750 & Tetradecanoic acid & $\mathrm{C}_{14} \mathrm{H}_{28} \mathrm{O}_{2}$ \\
\hline 23 & 35.878 & 1822 & 1,2-Benzenedicarboxylic acid, bis(2-methylpropyl) ester & $\mathrm{C}_{16} \mathrm{H}_{22} \mathrm{O}_{4}$ \\
\hline 24 & 41.694 & 1952 & n-Hexadecanoic acid & $\mathrm{C}_{16} \mathrm{H}_{32} \mathrm{O}_{2}$ \\
\hline 25 & 48.863 & 2111 & 9,12-Octadecadienoic acid, methyl ester & $\mathrm{C}_{19} \mathrm{H}_{34} \mathrm{O}_{2}$ \\
\hline 26 & 49.244 & 2120 & 9-Octadecenal, (Z)- & $\mathrm{C}_{18} \mathrm{H}_{34} \mathrm{O}$ \\
\hline
\end{tabular}

Note: The bold and italic fonts are used to refer to exclusive compounds. RT: Retention time. RI: Reflex index.

Table 7: The number and percentage of the common and exclusive hydrophilic volatile compounds identified from the six organs.

\begin{tabular}{|c|c|c|c|c|c|c|}
\hline Organ & Root & Stem & Stem Skin & Leaf & Flower & Fruit \\
\hline Total Compounds & 36 & 46 & 42 & 25 & 64 & 26 \\
\hline Common Compounds & \multicolumn{6}{|c|}{3} \\
\hline Percentage of Common Compounds & $8.3 \%$ & $6.5 \%$ & $7.1 \%$ & $12.0 \%$ & $4.7 \%$ & $11.5 \%$ \\
\hline Exclusive Compounds & 17 & 21 & 21 & 9 & 40 & 15 \\
\hline Percentage of Exclusive Compounds & $47.2 \%$ & $45.7 \%$ & $50.0 \%$ & $36.0 \%$ & $62.5 \%$ & $57.7 \%$ \\
\hline
\end{tabular}

practice of the traditional herbal medicine to treat the diseases using either the whole plant or part of the plants depending on which part contains the substances that can be used for therapeutic purposes.

Table 8 presents the number of overlapping compounds, overlapping percentage and overlapping index. The stem and stem skin share the largest number (15) of overlapping compounds. The overlapping percentage is calculated to be $32.6 \%$ for the stem and $35.7 \%$ for the stem skin. The smallest number (5) of overlapping compounds are found between root and fruit, leaf and fruit. The percentage of overlapping compounds between each of the two organs ranges from $10.9 \%$ to $44.0 \%$, which is relatively small, further demonstrating substantial component differences among the different organs. The overlapping index is used to reveal the similarity among the organs. Two organs share the same number of overlapping compounds, but the overlapping index could be different if the total number of the hydrophilic volatile compounds differs. The more total number of the hydrophilic volatile compounds, the less the percentage of the overlapping compounds and smaller the overlapping index.
That is why the average overlapping indices between the two organs is introduced to normalize the difference. In addition, total average overlapping indices is derived to calculate the mean of the average overlapping indices between each organ and the other five organs. Based on Table 8, the total average overlapping indices for each organ follows the order of stem $>$ stem skin $>$ root $>$ leaf $>$ flower $>$ fruit. The total average overlapping indices for the stem is the greatest at 3.056, indicating the stem share the maximum similarity with the plant. The total average overlapping indices for the fruit was the smallest at 1.090, indicating that the fruit share the minimum similarity with the plant. And there is not much difference in the average overlapping indices between fruit and the other five organs. Except fruit, the average overlapping indices between each of the two organs correlate well to their physical proximity. The root, stem and stem skin are the organs that the plant survive and grow, and their total average overlapping indices are greater than 2.5. The overlapping index differences among these three organs are small, and they share the most in common. As an evergreen plant, the leaf is symbiotically related to the plant 
Luosheng Huang (2021) Chemical Composition Similarity Relationships among the Various Organs of the Ilex Cornuta Lindl. \& Paxton Based on the Analysis of Hydrophilic Volatile Compounds

Table 8: The number of overlapping compounds, overlapping percentage and overlapping index.

\begin{tabular}{|c|c|c|c|c|c|c|c|}
\hline Organ 1 & Organ 2 & $\begin{array}{c}\text { Number of } \\
\text { overlapping } \\
\text { compounds }\end{array}$ & $\begin{array}{c}\text { Overlapping } \\
\text { percentage }\end{array}$ & $\begin{array}{c}\text { Overlapping index for } \\
\text { Organ } 1\end{array}$ & $\begin{array}{l}\text { Overlapping index } \\
\text { for Organ } 2\end{array}$ & $\begin{array}{c}\text { Average overlapping } \\
\text { indices between organ } \\
1 \text { and } 2 \\
\end{array}$ & $\begin{array}{c}\text { Total average } \\
\text { overlapping Indices }\end{array}$ \\
\hline \multirow{5}{*}{ Root } & Stem & 14 & $38.9 \%$ & 5.444 & 4.261 & 4.853 & \multirow{5}{*}{2.522} \\
\hline & Stem skin & 11 & $30.6 \%$ & 3.361 & 2.881 & 3.121 & \\
\hline & Leaf & 9 & $25.0 \%$ & 2.250 & 3.240 & 2.745 & \\
\hline & Flower & 7 & $19.4 \%$ & 1.361 & 0.766 & 1.064 & \\
\hline & Fruit & 5 & $13.9 \%$ & 0.694 & 0.962 & 0.828 & \\
\hline \multirow{5}{*}{ Stem } & Root & 14 & $30.4 \%$ & 4.261 & 5.444 & 4.853 & \multirow{5}{*}{3.056} \\
\hline & Stem skin & 15 & $32.6 \%$ & 4.891 & 5.357 & 5.124 & \\
\hline & Leaf & 9 & $19.6 \%$ & 1.761 & 3.240 & 2.501 & \\
\hline & Flower & 10 & $21.7 \%$ & 2.174 & 1.266 & 1.720 & \\
\hline & Fruit & 6 & $13.0 \%$ & 0.783 & 1.385 & 1.084 & \\
\hline \multirow{5}{*}{ Stem skin } & Root & 11 & $26.2 \%$ & 2.881 & 3.361 & 3.121 & \multirow{5}{*}{2.710} \\
\hline & Stem & 15 & $35.7 \%$ & 5.357 & 4.891 & 5.124 & \\
\hline & Leaf & 9 & $21.4 \%$ & 1.929 & 3.240 & 2.585 & \\
\hline & Flower & 10 & $21.4 \%$ & 1.929 & 1.266 & 1.598 & \\
\hline & Fruit & 6 & $14.3 \%$ & 0.857 & 1.385 & 1.121 & \\
\hline \multirow{5}{*}{ Leaf } & Root & 9 & $36.0 \%$ & 3.240 & 2.250 & 2.745 & \multirow{5}{*}{2.435} \\
\hline & Stem & 9 & $36.0 \%$ & 3.240 & 1.761 & 2.501 & \\
\hline & Stem skin & 9 & $36.0 \%$ & 3.240 & 1.929 & 2.585 & \\
\hline & Flower & 11 & $44.0 \%$ & 4.840 & 1.891 & 3.366 & \\
\hline & Fruit & 5 & $20.0 \%$ & 1.000 & 0.962 & 0.981 & \\
\hline \multirow{5}{*}{ Flower } & Root & 7 & $10.9 \%$ & 0.766 & 1.361 & 1.064 & \multirow{5}{*}{1.844} \\
\hline & Stem & 10 & $15.6 \%$ & 1.563 & 2.174 & 1.859 & \\
\hline & Stem skin & 10 & $14.1 \%$ & 1.266 & 1.929 & 1.598 & \\
\hline & Leaf & 11 & $17.2 \%$ & 1.891 & 4.840 & 3.366 & \\
\hline & Fruit & 7 & $10.9 \%$ & 0.766 & 1.885 & 1.326 & \\
\hline \multirow{5}{*}{ Fruit } & Root & 5 & $19.2 \%$ & 0.962 & 0.694 & 0.828 & \multirow{5}{*}{1.090} \\
\hline & Stem & 6 & $23.1 \%$ & 1.385 & 1.000 & 1.193 & \\
\hline & Stem skin & 6 & $23.1 \%$ & 1.385 & 0.857 & 1.121 & \\
\hline & Leaf & 5 & $19.2 \%$ & 0.962 & 1.000 & 0.981 & \\
\hline & Flower & 7 & $26.9 \%$ & 1.885 & 0.766 & 1.326 & \\
\hline
\end{tabular}

although the relationship between each leaf and the plant is cyclical, so the leaf is secondarily related to the plant. The flower and fruit are also cyclically related to the plant and have the most distant relationship. The leaf, flower and fruit are necessary but not survival organs for the growth of the plant. The relationship between the organs and the plant generated from the analysis of the hydrophilic volatile compounds is consistent with their biological function.

\section{Conclusion}

The root, stem, stem skin, leaf, flower and fruit of the Ilex cornuta Lindl. \& Paxton contain hydrophilic volatile compounds that are evenly distributed in the water phase of the various organs of the plant and can volatilize with water vapor. The number and type of hydrophilic volatile compounds vary from organ to organ. There is only a small number of common compounds among the six organs and the number of overlapping compounds between each of the two organs is also relatively small. In addition, there are large number of exclusive compounds from each organ. Therefore, it is possible to identify the plant through the assessment of the hydrophilic volatile compounds isolated from each individual organ.

In conclusion, we found that hydrophilic volatile metabolites are a class of natural products that are rarely investigated but constitute a significant part of the plant chemical composition. Chemical profiling of these secondary metabolites could provide a valuable tool for identification and authentication of the plant samples, as well as resolving taxonomic problems and understanding the chemically mediated biological phenomena.

\section{References}

1. Bickford D, Lohman DJ, Sodhi NS, Ng PKL, Meier R, et al. (2007) Cryptic species as a window on diversity and conservation. Trends Ecol Evol 22: 148-155. [crossref] 
2. Heinrichs J, Kreier HP, Feldberg K, Schmidt AR, Zhu RL, et al. (2011) Formalizing morphologically cryptic biological entities: New insights from DNA taxonomy, hybridization, and biogeography in the leafy liverwort Porella platyphylla (Jungermanniopsida, Porellales). Am J Bot 98: 1252-1262. [crossref]

3. Ludwiczuk A (2014) Fingerprinting of secondary metabolites of liverworts: chemosystematic approach. J of AOAC Int 97: 1234-1243.

4. Wink M (2003) Evolution of secondary metabolites from an ecological and molecular phylogenetic perspective. Phytochemistry 64: 3-19. [crossref]

5. Liu K, Abdullah AA, Huang M, Nishioka T, Altaf-Ul-Amin M, et al. (2017) Novel Approach to Classify Plants Based on Metabolite-Content Similarity. BioMed Res Int. doi: $10.1155 / 2017 / 5296729$

6. Ghaste M, Narduzzi L, Carlin S, Vrhovsek U, Shulaev V, et al. (2015) Chemical Composition of Volatile Aroma Metabolites and Their Glycosylated Precursors that Can Uniquely Differentiate Individual Grape Cultivars. Food Chem 188: 309-319. [crossref]

7. Peters K, Treutler H, Doll S, Kindt ASD, Hankemeier T, et al. (2019) Chemical Diversity and Classification of Secondary Metabolites in Nine Bryophyte Species. Metabolites 9: 222. [crossref]

8. Staszek D, Orlowska M, Rzepa J, Wrobel MS, Kowalska T (2014) Fingerprinting of the Volatile Fraction from Selected Thyme Species by Means of Headspace Gas Chromatography with Mass Spectrometric Detection. J of AOAC Int 97: 1250-1258. [crossref]
9. Tundis R, Peruzzi L, Menichini F (2014) Phytochemical and biological studies of Stachy Species in Relation to Chemotaxonomy: A Review. Phytochemistry 102: 7-39. [crossref]

10. Peñuelas J, Llusià J (1999) Seasonal emission of monoterpenes by the Mediterranean tree Quercus ilex in field conditions: Relations with photosynthetic rates, temperature and volatility. Physiol Plant 105: 641-647.

11. Llusia J, Penuelas J (2000) Seasonal patterns of terpene content and emission from seven mediterranean woody species in field conditions. Am J Bot 87: 133-140. [crossref]

12. Ormeo E, Goldstein A, Niinemets ü (2011) Extracting and trapping biogenic volatile organic compounds stored in plant species. TRAC-Trend Anal Chem 30: 978-989.

13. Claudia G, Roberta A, Daniela L, Giacomo T, Laura S, et al. (2018) Salvia verticillata: Linking glandular trichomes, volatiles and pollinators. Phytochemistry 155: 53-60.

14. Wei X, Song M, Chen C, Tong H, Liang G, et al. (2018) Juice volatile composition differences between Valencia orange and its mutant Rohde Red Valencia are associated with carotenoid profile differences. Food Chem 245: 223-232. [crossref]

15. Berlinck RGS, Monteiro AF, Bertonha AF, Bernardi DI, Gubiani JR, et al. (2019) Approaches for the isolation and identification of hydrophilic, light-sensitive, volatile and minor natural products. Nat Prod Rep 36: 981-1004. [crossref]

\section{Citation:}

Huang L (2021) Chemical Composition Similarity Relationships among the Various Organs of the Ilex Cornuta Lindl. \& Paxton Based on the Analysis of Hydrophilic Volatile Compounds. Internal Med Res Open J Volume 6(2): 1-10. 